\title{
Quantifying the contribution of multiple factors to land subsidence using artificial intelligence technology in Beijing, China
}

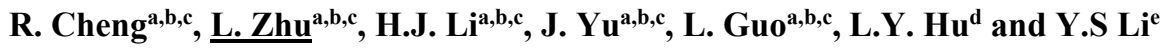 \\ ${ }^{a}$ College of Resources Environment and Tourism, Capital Normal University, Beijing 100048, China \\ ${ }^{b}$ Laboratory Cultivation Base of Environment Process and Digital Simulation, Beijing, P.R. China \\ ${ }^{c}$ Beijing Laboratory of Water Resources Security, Capital Normal University, Beijing 100048, China \\ ${ }^{d}$ Institute of Crustal Dynamics, China Earthquake Administration, Beijing 100085, China \\ e Beijing Earthquake Agency, Beijing 100080, China \\ Email:hi-zhulin@163.com
}

\begin{abstract}
More than 100 cities have experienced land subsidence in the world, but few studies have been conducted to quantitatively analyze the contribution rate of various factors to land subsidence. In this study, we established the BP-ANN-MIV (Back propagation-Artificial Intelligence Neural Network- Mean impact value ) model to quantify the contribution of multiple factors to land subsidence. The Chaobai River alluvial fan in Beijing, China is taken as the case study, where located the large scale well-fields and the international airport. The input parameters of the model include cumulative displacement detected by SBAS-InSAR technology, groundwater level change, the compressible layers thickness, building volume, road density data from 2014 to 2018 . The results show that the average displacement rate of the Chaobai River alluvial fan ranges from $-140 \mathrm{~mm} /$ year to $+21 \mathrm{~mm} /$ year. And most serious land subsidence occurred in the middle of the alluvial fan area. In the study area, the contribution rates of each factor are different in different years. The contribution rates of compressible layer thickness and groundwater level change are the largest, which are $28.59 \%-45.51 \%$ and $52.95 \%-69.57 \%$ respectively. The influence of road density and building volume is very small, about $2 \%$. The research is maybe helpful to managers for land subsidence prevention and control.
\end{abstract}

Keywords: $\quad$ Land subsidence, BP-ANN-MIV, Contrbution, InSAR 
Cheng et al., Quantifying the contribution of multiple factors to land subsidence using artificial intelligence technology in typical areas of Beijing, China

\section{INTRODUCTION}

Land subsidence is a common slow geohazard caused by the compression of soil layers, leading to a decrease in ground elevation. It has occurred in 150 regions in the world, posing a serious threat to human life and property. Beijing is a megacity with an acute water shortage and $2 / 3$ of water demand is supplied by groundwater(zhu et al., 2015). With the rapid urbanization, long-term overexploitation of groundwater has induced geological environmental problems such as the decline of groundwater level, poor water quality, and land subsidence, seriously threatening the normal development of the regional economy and society. It is of great significance to timely and effectively obtain the land subsidence information and quantify the contribution of multiple factors for land subsidence prevention and control.

Interferometric Synthetic Aperture Radar (InSAR) is a powerful tool widely used to map land subsidence. Compared with traditional geodetic techniques such as leveling, it has the advantages of low cost and high spatial-temporal resolutions. Small baseline subsets (SBAS) technology which can minimize the limitations (e.g., spatial and temporal decorrelation and atmospheric effects) is developed and demonstrated to be able to map the regional displacement of long-time series. It is successfully applied to monitor land subsidence worldwide.

At present, based on the temporal and spatial distribution of land subsidence obtained by InSAR technology, scholars have studied the qualitative relationship between land subsidence and influencing factors in Beijing. Studies by Zhu et al. (2015), Chen et al. (2015), Huang et al. (2016), Chen et al. (2016), Yang et al. (2018) show that relevant influencing factors (e.g., groundwater, compressible layer, building, and land use) have a certain correlation with the accelerated development of land subsidence. The other has quantified the effects of different factors on land subsidence. For example, Gao et al. (2019) introduced the cross wavelet method to determine the lag time of land subsidence affected by the change of groundwater level. Zhou et al (2019) utilized a random forest method to quantify the contribution of groundwater level, compressible layers, buildings and roads to land subsidence in Beijing plain. These studies mainly focused on qualitative analysis rather than quantification. And there is a lack of research on the quantification of the contribution of influencing factors to land subsidence considering the different hydrogeological conditions. So, it is necessary to carry out a more detailed quantitative research.

Given the above problems, the Chaobai river alluvial fan with a complex geological condition is used as the study area to quantitative the contribution rate of influencing factors to land subsidence. This paper (1) obtained the land subsidence map of the study area (2014.11-2018.07) using the SBAS method based on 43 Sentinel-1 A/B images; (2) analyzed the spatial and temporal distribution characteristics of land subsidence of by using spatial analysis technique; and (3) introduced the BP-MIV model to quantitatively determine the contribution rate of the compressible layer thickness, groundwater level change, dynamic and static load to land subsidence under the typical hydrogeological units.

\section{STUDY AREA}

Beijing is the capital of China with a population of more than 21 million in 2017. High urbanization induced the rapid construction of infrastructures and over exploration of groundwater resources. Chaibao alluvial fan is taken as the study area, where is the most important groundwater source area in Beijing. There are many large-scale wellfields supplying groundwater resources to Beijing city with an annual mining volume of about 600 million $\mathrm{m}^{3}$ (Figure 1). The study area is characterized by a warm temperate semi-humid monsoon continental climate, whose average annual temperature and precipitation are $11.7^{\circ} \mathrm{C}$ and $588 \mathrm{~mm}$ respectively (Chen et al. 2016). Rainfall is mainly concentrated from June to August., covering an area of about $11.67 \%$ of the total area of Beijing.

The Chaobai River alluvial fan has a complex geological environment, which upper part is

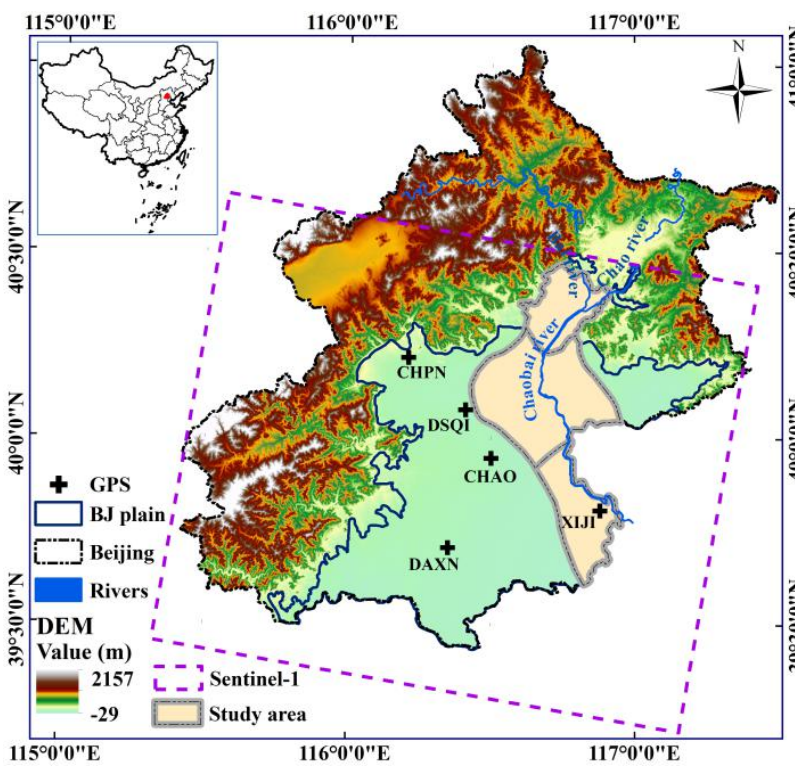

Figure 1. Location of study area. mainly composed of two rivers, Chao River and Bai River. The two rivers merge into a river in Chaobai River. 
Cheng et al., Quantifying the contribution of multiple factors to land subsidence using artificial intelligence technology in typical areas of Beijing, China

The sedimentary environment is complex. From the upstream to the downstream, the quaternary sediment thickness gradually increased. Corresponding to the Quaternary sedimentary law, the aquifer group gradually transits from the single aquifer to multi-layer aquifer from fan top to fan bottom (Zhu et al. 2015, Chen et al. 2016). And, from the surface to the deep, it can be roughly divided into four aquifers, and the lithology of aquifer gradually changes from a single gravel layer to fine sand and clay interbedding. The rich water property of the middle part in the study area is good, and the phreatic layer is easy to receive precipitation recharge. In this paper, the first three aquifers were selected for relevant research.

\section{DATASETS AND METHODOLOGY}

\subsection{Datasets}

The declining of groundwater level increased the pressure loading on deposites, which induced the displacement according to Terzaghi Principle. The compressible layer is the intrinsic factor inducing subsidence. Generally, the greater the thickness of the compressible layer and the lower the groundwater level, the more serious the land subsidence is. The surface loading, including the building and road loading, is another man-induced factor. The datasets include compressible layer thickness, groundwater level change, building volume change, road density change, and the cumulative land subsidence. It covers from 2014 to 2018, in which the South-to-North Water Transfer Project Middle Route starting operation.

This paper collected 43 Sentinel-1 A/B descent images of the IW model from November 2014 to July 2018 in order to obtain the surface displacement data. The data of groundwater level change is calculated from the contour of the groundwater level, which comes from the BEIJING WATER AUTHORITY. Because of the confidentiality of the original data, it cannot be listed here. The compressible layer thickness data are derived from Beijing land subsidence.

\subsection{Methodology}

In order to quantify the relationship between land subsidence and its influencing factors after South-to-North Water entering Beijing, this paper proposed the BP-ANN-MIV model to quantitatively analyze the impact of different factors on land subsidence. The flow chart is shown in Figure 2. Firstly, we collect the Sentinel-1 A/B images, groundwater level contours, compressible layer thickness contours, buildings, and roads. Then, we processed these data, obtained land subsidence by SBAS technology, discretized groundwater level contour into points and interpolated the groundwater level surface by ArcGIS technology, obtained the water level distribution covering the study area. According to the same method, the thickness distribution of the compressible layer is obtained. Finally, we assign all the information to the 500*500 meter grid, and input the grid data into the BP-ANN-MIV model to calculate the contribution rate of influencing factors in different years.

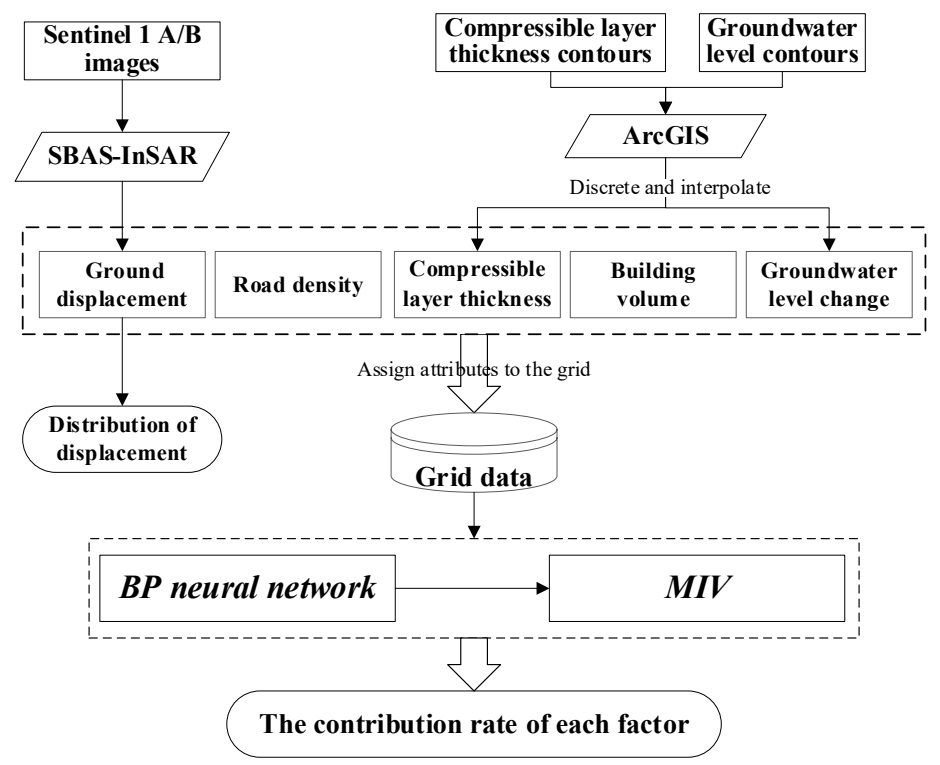

Figure. 2 Flow chart of data processing in this article. 
Cheng et al., Quantifying the contribution of multiple factors to land subsidence using artificial intelligence technology in typical areas of Beijing, China

\subsubsection{SBAS-InSAR technology}

InSAR time series analysis methods mainly include traditional persistent scatterer InSAR (PS-InSAR) and small baseline subset InSAR (SBAS- InSAR). The traditional PS method is mainly aimed at the stable radar scattering points composed of a single ground object and is not affected by the spatiotemporal decoherence. Compared with the traditional PS method, the small baseline set technology uses distributed scatterers (DS) to improve the coherence point density to a certain extent. Therefore, this method is selected to extract time-series information of land subsidence.

\subsubsection{BP (back propagation) -ANN (artificial intelligence neural network) -MIV (mean impact value) model}

BP-ANN is a kind of feed-forward neural network that has the ability to describe any complex nonlinear implicit function at an arbitrary precision (K and O, 1992), which is essentially a BP learning algorithm of error backpropagation. It can correct the connection weight and threshold error of each layer of the network

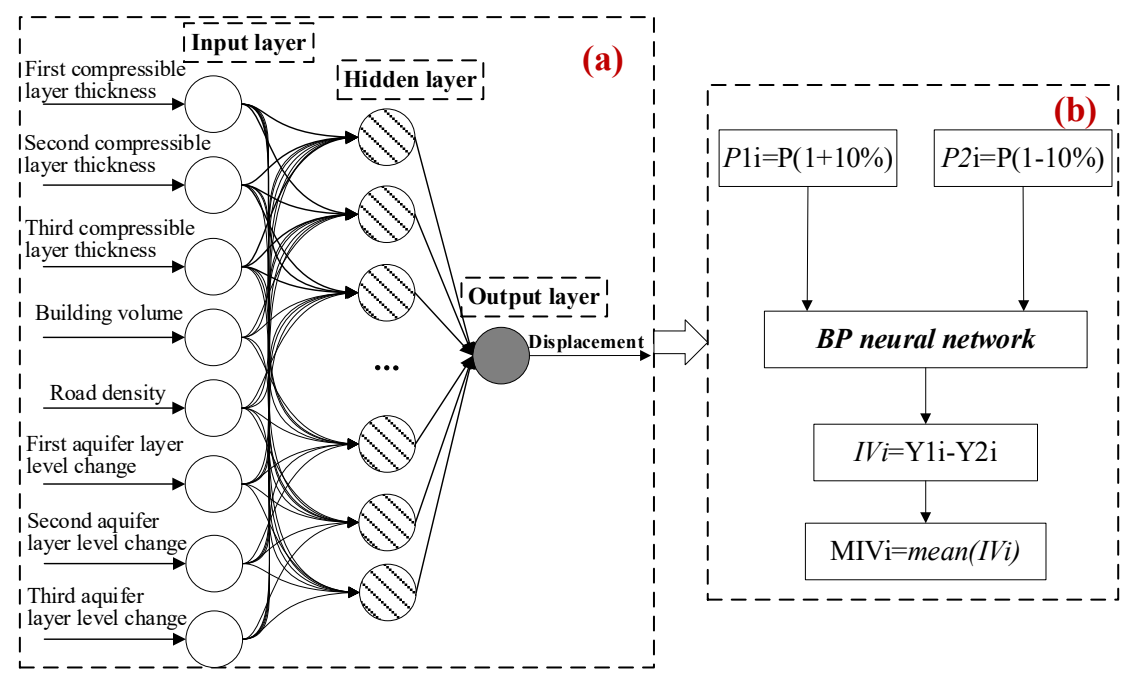

Figure. 3 Structural sketch of MIV-BP-ANN.(a) is the principle of BP neural network. (b) is the flow of MIV

from the back to the front repeatedly until the minimum error between the model output and the actual value. Therefore, this paper chose the threelayer BP neural network with a single hidden layer as the base model to calculate the contribution rate of multiple factors.

The structure diagram of the BP-ANN-MIV can be illustrated in Figure 3. The MIV value can reflect the change of weight matrix in neural networks and assess the influence of input neurons on output neurons (Zhang et al.,2016). The process is as follows:(1) The BP neural network is trained with the original data, and the training is stopped after the accuracy reaches the standard, and the network is saved. (2) On the basis of the original value of a variable, add or subtract $10 \%$ to form $\mathrm{P} 1$ and $\mathrm{P} 2$, while the other variables remain unchanged. (3) P1 and P2 were input into the trained BP neural network simulation, and two simulation results, Y1 and Y2, respectively, were obtained. (4) The impact value (IV) of this variable is the difference between Y1 and Y2, which represents the effect of changing this variable on the output. (5) The IVs were averaged by the number of observations to obtain the MIV of the independent variable on the dependent variable. The MIV of each independent variable was calculated according to the above steps. The MIV values of the generated variables change when the neural network is trained each time. Therefore, repeat (1) - (5) K times in order to obtain reliable MIV values. Finally, the relative contribution rate of $i$ th independent variable to the dependent variable was calculated according to $\operatorname{Eq}(1)$.

$$
\partial_{i}=\frac{\left|\frac{1}{k} \sum_{j=1}^{k} M I V_{i}\right|}{\sum_{i}^{m}\left|\frac{1}{k} \sum_{j=1}^{k} M I V_{i}\right|}
$$

Where, $\partial_{i}$ is the contribution rate of variable $i, m$ is the total number of variables, that is $8, k$ is the number of repeated training network. After repeated tests, in order to ensure the running speed and accuracy of the results, 1000 times are selected in this paper. 
Cheng et al., Quantifying the contribution of multiple factors to land subsidence using artificial intelligence technology in typical areas of Beijing, China

\section{RESULTS AND DISCUSSION}

\subsection{Distribution of land subsidence}

The distribution of land subsidence in the Chaobai River alluvial fan was obtained by using SBAS technology. High-precision GPS monitoring data was used to validate the outcomes deduced from InSAR technology. Five highprecision GPS monitoring stations from 2014 to 2018 were selected as verification points, and the GPS monitoring data (east-west, north-south, vertical) were projected to the line of sight (LOS) of InSAR monitoring. The average value of PS points in the $150 \mathrm{~m}$ buffer zone of the monitoring station was selected and compared with those of the corresponding GPS monitoring stations. The minimum absolute error is $-1.08 \mathrm{~mm} /$ year, and the standard deviation is $5.98 \mathrm{~mm} /$ year. The correlation coefficient between GPS monitoring data and InSAR monitoring data is up to 0.98 . The verification results show that the monitoring results of InSAR are in good agreement with those of GPS, which indicates the reliability of the monitoring results of InSAR.

Figure 4 shows the distribution of the displacement rate in the study area, which reflects the development of land subsidence in the monitoring period. From November 2014 to July 2018, the maximum displacement rate was about $-14 \mathrm{o} \mathrm{mm} / \mathrm{y}$, was located in the transitional area between the middle and lower parts of the Chaobai River alluvial fan, and land subsidence occurred in more than $47.29 \%$ of the study area. Among them, the area with a settlement rate exceeding $50 \mathrm{~mm} /$ year was $46.51 \mathrm{~km}^{2}$, accounting for $2.42 \%$ of the total area of the study area. In

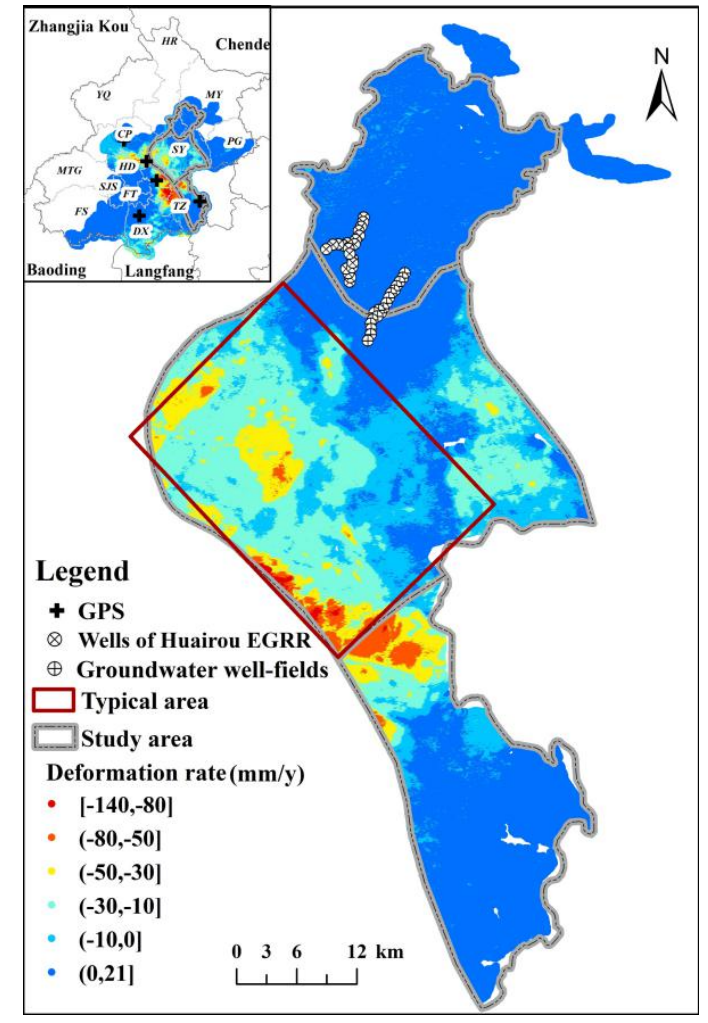

Figure 4. Distribution map of deformation rate in study area from 2014.11 to 2018.07 . the north part of the study area, the subsidence can be negligible (marked with dark blue). High subsidence rate with the value larger than $50 \mathrm{~mm} / \mathrm{y}$ mainly occurred in the middle part of the Chaobai alluvial fan (marked with yellow). And, at the lower part of the fan margin, there is almost no subsidence as well (marked with dark blue).

\subsection{The contribution of multiple factors to surface displacement}

BP-ANN-MIV artificial intelligence model is constructed with compressible layer thickness, groundwater level change, road density and building volume as input neurons and cumulative displacement as the output neuron. And the calculation contribution rate of each factor under different years was shown in Table 1. It should be pointed out that in order to discuss the role of groundwater level change as a whole, the contribution rates of three aquifers are added together, and so is the thickness of compressible aquifers. It can be found that groundwater level change and compressible layer thickness have the greatest contribution to land subsidence. The contribution rate range of groundwater level change is $52.95 \%$ to $69.57 \%$, and the contribution rate range of compressible layer thickness is $28.59 \%$ to $45.51 \%$. And the contribution rates of building volume and road density are small, ranging from $0 \%$ to $2 \%$.

Table 1. The contribution rate of each dependent in the special area during this period.

\begin{tabular}{llllll}
\hline ID & Dependent variables & $2015(\%)$ & $2016(\%)$ & $2017(\%)$ & Range (\%) \\
\hline 1 & Compressible layer thickness & $\mathbf{4 5 . 5 1}$ & $\mathbf{2 8 . 5 9}$ & $\mathbf{3 5 . 6 9}$ & $28.59-45.51$ \\
2 & Groundwater level & $\mathbf{5 2 . 9 5}$ & $\mathbf{6 9 . 5 7}$ & $\mathbf{6 0 . 8 4}$ & $52.95-69.57$ \\
3 & Building volume & 0.98 & 0.65 & 1.41 & $0.65-1.41$ \\
4 & Road density & 0.55 & 1.18 & 2.06 & $0.55-2.06$ \\
\hline
\end{tabular}


Cheng et al., Quantifying the contribution of multiple factors to land subsidence using artificial intelligence technology in typical areas of Beijing, China

Affected by human activities and natural weather, the influencing factors that groundwater level, road density, and building volume will change to some extent. External human activities are intense, and the corresponding contribution rate range of the influencing factor affected by such human activities will change greatly. Influenced by the South-to-North Water Transfer Project and precipitation density, the groundwater level varies greatly in different years, so the contribution rate of the calculated groundwater level varies greatly. For example, the amount of surface water, groundwater resources and precipitation in 2016 is the largest in the study period, with 1.401 billion $\mathrm{m}^{3}, 2.105$ billion $\mathrm{m}^{3}$ and $660 \mathrm{~mm}$ respectively. Therefore, it is speculated that this may be the reason why the contribution rate of groundwater level change in 2016 is greater than that in 2017. In addition, the compressible layer thickness will hardly change in thousands years. It is presumed that the contribution rate change of the compressible layer mainly is influenced by the change of groundwater contribution rate. On the contrary, building volume and road density changed changed slightly in different years, so the range of change in their contribution rate is very small.

Therefore, it can be inferred when the number of years involved in the calculation is large enough, a basic accurate range can be obtained. In addition, the analysis of the reasons for the change of factor contribution rate needs to be combined with the actual situation of that year. For example, to analyze the impact of groundwater in one year, it is necessary to combine the data of groundwater exploitation, recharge and exploitation depth in that year. Therefore, in order to obtain a more accurate contribution rate of influencing factors, it is necessary to collect more abundant data.

\section{CONCLUSION}

Based on the Sentinel-1 A/B image, the surface displacement information of the Chaobai River alluvial fan area is obtained by SBAS-InSAR technology. The accuracy of monitoring results is verified by GPS data, and its spatial and temporal distribution is analyzed by ArcGIS spatial analysis technology. The results show that the correlation coefficient between InSAR and GPS monitoring results is 0.98 , which is reliable. Compared with the alluvial fan top, the subsidence of the middle and lower parts of the study area is more serious, and it can be found that the land subsidence is serious at the junction of Huairou sub-region and Xiji sub-region where different hydrogeological units transit.

Based on the BP neural network, the BP-ANN-MIV model with cumulative displacement as the dependent variable, compressible layer thickness, groundwater level change, road density and building volume as independent variables was established. The contribution rates of factors to surface displacement from 2015 to 2017 are obtained. It is found that the most important contributing factors are compressible layer thickness $(28.59 \%-45.51 \%)$ and groundwater level change $(52.95 \%-69.57 \%)$. The contribution rates of building volume and road density are small, basically floating around $1 \%$. The research is maybe helpful to managers for land subsidence prevention and control considering the different hydrogeological conditions.

\section{ACKNOWLEDGMENTS}

The research activities were supported by the Chinese National Natural Science Foundation (No. 41771455), Young Yanjing Scholar Program, the Capacity Building for Sci-Tech Innovation- Fundamental Scientific Research Funds (No. 025185305000/191).

\section{REFERENCES}

Zhu L, Gong H, Li X, et al. Land subsidence due to groundwater withdrawal in the northern Beijing plain, China[J]. Engineering Geology, 2015, 193:243-255.

Chen, B.B., Gong, H.L, Li, X.J. 2015. Spatial correlation between land subsidence and urbanization in Beijing, China. Natural Hazards, 75(3): 2637-2652.

Huang M H, Bürgmann, Roland, Hu J C. Fifteen years of surface deformation in Western Taiwan: Insight from SAR interferometry[J]. Tectonophysics, 2016: S0040195116001189.

M Chen, Tomás Roberto, Zhenhong L, et al. Imaging Land Subsidence Induced by Groundwater Extraction in Beijing (China) Using Satellite Radar Interferometry[J]. Remote Sensing, 2016, 8(6):468-.

Qin Y, Yinghai K, Dongyi Z, et al. Multi-Scale Analysis of the Relationship between Land Subsidence and Buildings: A Case Study in an Eastern Beijing Urban Area Using the PS-InSAR Technique[J]. Remote Sensing, 2018, 10(7):1006-.

M Gao., H Gong., X Li., B Chen., et al. Land subsidence and ground fissures in Beijing Capital International Airport (BCIA): Evidence from Quasi-PS InSAR analysis[J]. Remote Sensing, 2019, 11(12):1466.

C Zhou, H Gong., B Chen., et al. Quantifying the contribution of multiple factors to land subsidence in the Beijing Plain, China with machine learning technology[J]. Geomorphology, 2019, 335:48-61. 
Cheng et al., Quantifying the contribution of multiple factors to land subsidence using artificial intelligence technology in typical areas of Beijing, China

Bennett K, Mangasarian O L. Robust linear programming discrimination of two linearly inseparable sets[J]. Optimization Methods and Software, 1992, 1(1):23-34.

Z L. Zhang, J.G. Yang, Narrow density fraction prediction of coarse coal by image analysis and MIV-SVM, Int. J. Oil Gas Coal Technol. 11 (3) (2016) 279-289. 